\title{
Los grupos cooperativos. Las especialidades del régimen de consolidación fiscal
}

(Cooperative groups. The specialties within the tax consolidation regime)

\author{
Pedro J. González-Perabá Miralles ${ }^{1}$ \\ Universidad de Deusto (España)
}

Sumario: 1. Introducción. El principio de la intercooperación. 2. Los grupos de sociedades y los grupos cooperativos en la legislación mercantil. 2.1 Los grupos de sociedades en el Código de Comercio. 2.2 Los grupos cooperativos. 3. La fiscalidad de los grupos cooperativos. 3.1 El régimen fiscal especial de las cooperativas. 3.2 El régimen de consolidación fiscal en los grupos cooperativos. 3.2.1 Definición de grupo de sociedades cooperativas. 3.2.2 La determinación de la base/ cuota consolidada. 4. Conclusiones.

Summary: 1. Introduction. The principle of intercooperation. 2. Company groups and cooperative groups in commercial law. 2.1 Company groups in the Commercial Code. 2.2 Cooperative groups. 3. The Taxation of cooperative groups. 3.1 The special tax regime for cooperatives. 3.2 The tax consolidation regime in cooperative groups. 3.2.1 Definition of cooperative groups. 3.2.2 Determination of the consolidated base / quota. 4. Conclusions.

Resumen: Los grupos cooperativos constituyen una de las manifestaciones del principio de intercooperación. Si los grupos de sociedades se basan en la idea del control que una sociedad ejerce sobre otra u otras, los grupos cooperativos se basan en la existencia de una unidad de dirección por parte de la entidad cabeza del grupo, que deriva de un acuerdo de voluntades entre las cooperativas que lo integran. Esta diferente naturaleza da lugar a importantes especialidades en el régimen jurídico de los grupos cooperativos, que se presentan tanto en su regulación mercantil como tributaria.

En este artículo analizaremos las especialidades que presenta el régimen de consolidación fiscal de los grupos cooperativos, tanto en la normativa de Territorio Común como de los Territorios Históricos del País Vasco, frente al ré-

1 Email: p.gonzalez.peraba@opendeusto.es 
gimen de consolidación fiscal contenido en la normativa general del Impuesto sobre Sociedades.

Palabras clave: intercooperación, cooperativas, grupos cooperativos, grupos de sociedades, consolidación fiscal.

Abstract: Cooperative groups are one of the manifestations of the principle of intercooperation. If company groups are based on the idea of the control that one company exercises over one or others, cooperative groups are based on the existence of a management unit by the head of the group, which derives from an agreement of the cooperatives that constitute it. This different nature gives rise to important specialties in the legal regime of cooperative groups, which are present both in their commercial and tax regulations.

In this article, we will analyse the specialties that the tax consolidation regime of cooperative groups have, both in the regulations of the Common Territory and the Historical Territories of the Basque Country, as opposed to the fiscal consolidation regime include in the general regulations on Corporation Tax.

Keywords: intercooperation, cooperatives, cooperative groups, corporate groups, tax consolidation. 


\section{Introducción. El principio de la intercooperación}

El cooperativismo surgió en la ciudad de Manchester, en el contexto de la Revolución Industrial, que tuvo lugar durante el siglo XIX. Fue en el año 1844 cuando se constituyó la que habitualmente se considera como primera experiencia del movimiento cooperativo: Rochdale Equitable Pioneers Society. Era una cooperativa de consumo, constituida por 28 personas, 27 hombres y una mujer, que proporcionó a sus miembros alimentos básicos a precios asequibles.

Las ideas en las que se basaron estos primeros pasos en el movimiento cooperativista fueron, posteriormente, fuente de inspiración de la Alianza Cooperativa Internacional $(\mathrm{ACl})$, organización creada en Londres en el año 1895, que estableció los principios en los que debía basarse este movimiento, y que han sido objeto de sucesivas actualizaciones. La última de estas actualizaciones tuvo lugar, con la aprobación de la Declaración de Principios de la $\mathrm{ACl}$, en un congreso celebrado en Manchester en 1995.

Durante este congreso se establecieron los siguientes siete principios, que constituyen la base del movimiento cooperativo mundial en la actualidad:

1. Asociación voluntaria y abierta

2. Control democrático por los miembros

3. Participación económica de los miembros

4. Autonomía e independencia

5. Compromiso por la educación sobre la ética y la práctica cooperativa

6. Cooperación entre cooperativas

7. Interés por el desarrollo sostenible de sus comunidades.

El sexto principio, el de la «cooperación entre cooperativas» o «intercooperación», debe ser entendido en el sentido de que «las cooperativas sirven a sus miembros más eficazmente y fortalecen el movimiento cooperativo trabajando conjuntamente mediante estructuras locales, nacionales, regionales e internacionales». Así, de la misma manera que desde los años setenta del siglo xx se ha generalizado la globalización de la economía, siendo habitual la creación de grandes grupos de sociedades transnacionales, en el movimiento cooperativo, este fenómeno tiene su manifestación a través de la creación de cooperativas de segundo grado y grupos cooperativos.

Para Iñigo Nagore (Nagore 2000, 117), la integración cooperativa tiene un doble objetivo: 
1. El reto de la «crisis del medio», es decir, evitar que las empresas cooperativas sean absorbidas por el sector capitalista.

2. Mejorar la autoayuda que se realiza internamente en cada cooperativa, imitando los procedimientos utilizados por las empresas capitalistas para conseguir un mayor tamaño, pero sin que esto suponga un abandono de los principios cooperativos.

Como señala Aitor Bengoetxea Alkorta (Bengoetxea Alkorta 2016, 9), por encima de las cooperativas de primer grado, el principio de intercooperación da lugar habitualmente a la constitución de cooperativas de segundo grado y de grupos cooperativos.

Las cooperativas de segundo grado son estructuras asociativas, cuyo objeto es promover, coordinar y reforzar la actividad económica de las entidades miembros, que podrán ser cooperativas de primer grado, así como personas socias de trabajo, y cualesquiera otras entidades y personas jurídicas de naturaleza pública o privada, siempre que exista la necesaria convergencia de intereses o necesidades y que el estatuto de la cooperativa de segundo grado no lo impida.

Por su parte los grupos cooperativos, constituyen una figura más limitada que las cooperativas de segundo grado, pues integran un conjunto de varias sociedades cooperativas y la entidad cabeza de grupo, que emitirá instrucciones de obligado cumplimiento para las cooperativas agrupadas, de forma que se genera una unidad de decisión en el ámbito de las facultades atribuidas a dicha entidad.

Frente a las cooperativas de segundo grado, en las que pueden integrarse personas físicas y personas jurídicas privadas o públicas con una forma legal diferente a la de las cooperativas, en el grupo cooperativo la única entidad que puede adoptar una forma distinta a la cooperativa sería la entidad cabeza del grupo.

\section{Los grupos de sociedades y los grupos cooperativos en la legislación mercantil}

\subsection{Los grupos de sociedades en el Código de Comercio}

Para acometer el estudio de los grupos cooperativos es necesario que nos detengamos, aunque sea brevemente, en el estudio de las diferencias existentes entre estos y los grupos de sociedades regulados en el artículo 42 del Código de Comercio (en adelante CCo).

A día de hoy, no existe en nuestra legislación mercantil una definición de grupo de sociedades, que tenga, de forma explícita, un carác- 
ter general. Sin embargo, la mayoría de la doctrina ${ }^{2}$ está de acuerdo en considerar que el artículo 42 CCo contiene una definición general aplicable a todos los ámbitos del derecho mercantil, debido, entre otros motivos, a la referencia que a dicho artículo se ha ido introduciendo en otras leyes mercantiles, para determinar qué se entendía por grupo de sociedades a los efectos de dichas leyes. Así, actualmente se remiten a este artículo la Ley 24/1988, de 28 de julio, del Mercado de Valores (BOE núm. 181, de 29 de julio de 1988), la Ley de Sociedades de Capital, aprobada por Real Decreto Legislativo 1/2010, de 2 de julio (BOE núm. 161, de 3 de julio de 2010), y la Ley 38/2011, de 10 de octubre, de reforma de la Ley 22/2003, de 9 de julio, Concursal (BOE núm. 245, de 11 de octubre de 2011).

Tradicionalmente se ha discutido si el elemento esencial que debía concurrir para la existencia de un grupo de sociedades era la presencia de una dirección económica unitaria, es decir, una unidad de decisión, o, en cambio, la existencia de una situación de control de una sociedad, denominada sociedad dominante, sobre el resto, denominadas sociedades dependientes o subordinadas.

José Miguel Embid Irujo (Embid Irujo 2012,4-5) plantea dudas en torno a cuál de estos dos elementos debe ser considerado como esencial para la configuración del concepto de grupo o si ambos deben estar presentes en todo caso para poder aceptar la existencia de un grupo. Aunque este autor considera que la dirección unitaria es un elemento esencial del grupo de sociedades, admite que, sobre todo en el ámbito legislativo, se ha ido imponiendo la idea de que había que delimitar el concepto de grupo sobre la base de la existencia de una situación de control, en lugar de sobre la concurrencia de una dirección unitaria, por considerar esta como una realidad propia del mundo empresarial, difícil de trasladar a la legislación mercantil.

Estas dudas doctrinales se han reflejado en las sucesivas redacciones del artículo 42 del CCo. Fue el artículo 106 de la Ley 62/2003, de 30 de diciembre, de medidas fiscales, administrativas y del orden social (BOE núm. 313, de 31 de diciembre de 2013), el que introdujo en el Código de Comercio, por primera vez, una definición de grupo, al señalar que "existe un grupo cuando varias sociedades constituyan una unidad de decisión». A continuación, se establecía que «se presumirá que existe dicha unidad de decisión», cuando una sociedad mercantil

2 Entre otros: Rojo Fernández-Río 1996; López Aparcero 2012; García Orejudo 2012, 69-78; Castro de Luna 2015; Ramos Muñoz 2015, 840-859. 
que, siendo socia de otra sociedad, se encuentre en alguna de las siguientes situaciones:

1. Posea la mayoría de los derechos de voto.

2. Tenga la facultad de nombrar o de destituir a la mayoría de los miembros del órgano de administración.

3. Pueda disponer de la mayoría de los derechos de voto, en virtud de acuerdos con otros socios.

4. Haya nombrado, exclusivamente con sus votos, a la mayoría de los miembros del órgano de administración, que desempeñen su cargo en el momento en que deban formularse las cuentas consolidadas y durante los dos ejercicios inmediatamente anteriores.

Y para completar esta lista se añadió el párrafo 2, según el cual «se presumirá igualmente que existe unidad de decisión cuando, por cualesquiera otros medios, una o varias sociedades se hallen bajo dirección única. En particular, cuando la mayoría de los miembros del órgano de administración de la sociedad dominada sean miembros del órgano de administración o altos directivos de la sociedad dominante o de otra dominada por esta».

Frente a esta redacción del año 2003, el contenido actual del artículo 42 CCo procede de la Ley 16/2007, de 4 de julio, de reforma y adaptación de la legislación mercantil en materia contable para su armonización internacional, con base en la normativa de la Unión Europea (BOE núm. 160, de 5 de julio de 2007). En esta reforma se abandonó toda referencia a la unidad de decisión sustituyéndola por el control como elemento definitorio de los grupos de sociedades. Así, actualmente se establece que «existe un grupo cuando una sociedad ostente o pueda ostentar, directa o indirectamente, el control de otra u otras». A continuación, se sustituye la expresión "se presumirá que existe unidad de decisión...» por la de que "se presumirá que existe control...», pero manteniendo la relación de los cuatro supuestos, anteriormente indicados, en los que, en la redacción anterior, se presumía la existencia de una unidad de decisión.

\subsection{Los grupos cooperativos}

Como hemos indicado anteriormente, la mayoría de las leyes mercantiles contienen una referencia al artículo 42 CCo para definir los grupos de sociedades a los efectos de dichas leyes. Así ocurre en la Ley del Mercado de Valores, la Ley de Sociedades de Capital, y la Ley Concursal. 
Dicho esto, debemos destacar como excepción a esta remisión general, la regulación que se recoge en la normativa sobre cooperativas, que presenta una definición de grupo con importantes diferencias respecto al artículo 42 CCo. Es necesario que nos detengamos en el estudio de estas diferencias entre grupos de sociedades y grupos cooperativos, pues dichas diferencias van a tener una gran transcendencia en la regulación de los requisitos subjetivos que deben cumplir las entidades integrantes del grupo de cooperativas para que se pueda aplicar el régimen de consolidación fiscal previsto en el Impuesto sobre Sociedades (en adelante ISOC), tal y como expondremos posteriormente.

El artículo 78 de la Ley 27/1999, de 16 de julio, de Cooperativas establece que «se entiende por grupo cooperativo, a los efectos de esta Ley, el conjunto formado por varias sociedades cooperativas, cualquiera que sea su clase, y la entidad cabeza de grupo que ejercita facultades o emite instrucciones de obligado cumplimiento para las cooperativas agrupadas, de forma que se produce una unidad de decisión en el ámbito de dichas facultades» ${ }^{3}$.

A continuación, el artículo 78 de la LC establece que la incorporación al grupo requerirá del acuerdo inicial de cada una de las entidades. También es necesario que los compromisos generales asumidos ante el grupo se formalicen por escrito, que deberá elevarse a escritura pública, comprendiendo como mínimo el acuerdo de duración, cuando sea limitado en el tiempo, el procedimiento para su modificación, así como para la separación de alguna sociedad cooperativa y las facultades cuyo ejercicio se acuerda atribuir a la entidad cabeza del grupo.

En relación al contenido de este artículo Carlos Vargas Vasserot, Enrique Gadea Soler y Fernando Sacristán Bergia (Vargas Vasserot, Gadea Soler y Sacristán Bergia 2017, 224), recuerdan que en los grupos cooperativos deberá regir, entre otros principios, el principio de libre adhesión. La consecuencia de este principio es que la pertenencia al grupo va a estar siempre supeditada a la voluntad de las cooperativas

3 No contienen regulación propia de los grupos cooperativos sino que se remiten a la legislación estatal: la Ley 5/1998, de 18 de diciembre, de Cooperativas de Galicia (Boletín Oficial de Galicia núm. 251, de 30 de diciembre de 1998), la Ley 4/1999, de 30 de marzo, de Cooperativas de la Comunidad de Madrid (Boletín Oficial de la Comunidad de Madrid núm. 87, de 14 de abril, de 1999), la Ley 4/2001, de 2 de julio, de Cooperativas de La Rioja (Boletín Oficial de la Rioja núm. 82, de 10 de julio de 2001), la Ley 11/2010, de 4 de noviembre, de Cooperativas de Castilla-La Mancha (BOE núm. 37, de 12 de febrero de 2011), y el Decreto Legislativo 2/2014, de 29 de agosto, del Gobierno de Aragón, por el que se aprueba el texto refundido de la Ley de Cooperativas de Aragón (Boletín Oficial de Aragón núm. 176, de 9 de septiembre de 2014). 
de base, que en cualquier momento podrán separarse del grupo e incluso decidir la expulsión de uno de sus miembros.

Idéntica definición a la contenida en el artículo 78 se recoge en la legislación autonómica sobre cooperativas de Murcia, Navarra, Asturias, Cantabria, Valencia y Extremadura ${ }^{4}$.

Por su parte, el artículo 142 de la Ley 1/2003, de 20 de marzo, de Cooperativas de las Islas Baleares (Boletín Oficial de las Islas Baleares núm. 42, de 29 de marzo de 2003) y el artículo 128 del Proyecto de Ley de Cooperativas de Canarias (Boletín Oficial de Canarias núm. 34, de 19 de febrero de 2020), contienen un concepto, diferente en cuanto a su redacción, pero similar en cuanto a su contenido, al del artículo 78 de la Ley 27/1999, al señalar que «se entiende por grupo cooperativo a los efectos de esta ley el conjunto formado por diversas sociedades cooperativas de cualquier clase, que tiene por objeto la definición de políticas empresariales y su control, la planificación estratégica de la actividad de los socios y la gestión de los recursos y actividades comunes. Los estatutos del grupo cooperativo determinarán las facultades de administración y gestión que deberá tener su entidad cabeza de grupo, las instrucciones son de cumplimiento obligado para las cooperativas agrupadas, de manera que se produce una unidad de decisión en el ámbito de las facultades mencionadas».

Por otro lado, el artículo 109 de la Ley de Ley 14/2011, de 23 de diciembre, de Sociedades Cooperativas Andaluzas (Boletín Oficial de la Junta de Andalucía núm. 255, de 31 de diciembre de 2011), diferencia entre grupos de cooperativas propios e impropios. Señala que «grupo cooperativo propio es aquel en el que existe una sociedad cabeza de grupo que ejercita facultades o emite instrucciones de obligado cumplimiento para el grupo. Dicha sociedad cabeza de grupo podrá tener naturaleza cooperativa o no, si bien, en este último caso, los miembros

${ }^{4}$ En el artículo 134 de la Ley 8/2006, de 16 de noviembre, de Sociedades Cooperativas de la Región de Murcia (Boletín Oficial de la Región de Murcia núm. 282, de 7 de diciembre de 2006); en el artículo 82 de Ley Foral 14/2006, de 11 de diciembre, de Cooperativas de Navarra (Boletín Oficial de Navarra núm. 149, de 13 de diciembre de 2006); en el artículo 134 de la Ley 4/2010, de 29 de junio, de Cooperativas de la Comunidad Autónoma del Principado de Asturias (Boletín Oficial del Principado de Asturias núm. 160, de 12 de julio de 2010); en el artículo 132 de la Ley 6/2013, de 6 de noviembre, de Cooperativas de Cantabria (BOE núm. 284, de 27 de noviembre de 2013); en el artículo 103 del Decreto Legislativo 2/2015, de 15 de mayo, del Consell, por el que aprueba el texto refundido de la Ley de Cooperativas de la Comunidad Valenciana (Diario Oficial de la Generalitat Valenciana núm. 7529, de 20 de mayo de 2015); y en el artículo 135 de la Ley 9/2018, de 30 de octubre, de Sociedades Cooperativas de Extremadura (Diario Oficial de Extremadura núm. 213, de 2 de noviembre de 2018). 
del grupo habrán de ser sociedades cooperativas en su mayoría» y será "grupo cooperativo impropio aquel en el que sus miembros, que habrán de ser sociedades cooperativas en su mayoría, se articulan en un plano de igualdad, funcionando sobre la base de un principio de coordinación».

Por último, debemos destacar que la legislación del País Vasco, recogida en la Ley 11/2019, de 20 de diciembre, de Cooperativas de Euskadi (Boletín Oficial del País Vasco núm. 247, de 30 de diciembre de 2019), se refiere al grupo cooperativo en el párrafo 1 de su artículo 154, en idénticos términos que el artículo 78 de la Ley 27/1999. Pero a continuación, el párrafo 2 del mismo artículo 154, contiene una clasificación parecida a la de la Ley de Sociedades Cooperativas Andaluzas, aunque, en lugar de diferenciar entre grupos propios e impropios, distingue entre los grupos cooperativos por integración y grupos cooperativos por colaboración, en función del grado de integración económica. Se entiende incluidos en el primer grupo aquellos en los que exista una dirección general común y un nivel de centralización de las facultades de gestión económica que permitan entender que estamos ante una verdadera unidad económica. En otro caso, cuando el nivel de integración no permita afirmar que estamos ante una unidad económica, se entenderá que estamos ante un grupo por colaboración.

Parece así, tal y como señalan Rosalía Alfonso Sánchez y Mercedes Sánchez Ruiz (Alfonso Sánchez y Sánchez Ruiz 2007, 11-13), que el concepto de grupo cooperativo, a diferencia del artículo 42 CCo, no se basa en la existencia de control por parte de la entidad cabeza del grupo, sino en la existencia de una unidad de dirección o decisión. De hecho, en los grupos cooperativos la sumisión a la dirección unitaria no resulta de una dominación de la entidad cabeza del grupo sobre las demás, como ocurre en los grupos del artículo 42 CCo, sino que deviene de un acuerdo de voluntades en virtud del cual se realiza una transferencia de competencias desde las sociedades agrupadas, al núcleo dirigente de grupo. Normalmente los grupos cooperativos serán el fruto de la autonomía de la voluntad y por tanto el elemento esencial en ellos será el contrato creador del grupo.

Esta importante diferencia va a ser esencial al regular los requisitos que deben cumplir las cooperativas integrantes de un grupo para poder aplicar el régimen de consolidación fiscal del ISOC, pues no existiendo en los grupos cooperativos un control por parte de la entidad cabeza del grupo sobre sus miembros, sino más bien al contrario, no tendría sentido exigir, en los grupos cooperativos, los porcentajes de participación establecidos en la normativa del ISOC para la aplicación del régimen de consolidación. 


\section{La fiscalidad de los grupos cooperativos}

\subsection{El régimen fiscal especial de las cooperativas}

Antes de adentrarnos en el estudio de las especialidades del régimen de consolidación fiscal, previstas para los grupos cooperativos, la cuestión previa que debemos plantearnos es la de por qué las cooperativas, tanto de primer como de segundo grado, necesitan recibir un tratamiento tributario especial en nuestra normativa, diferente al previsto con carácter general para el resto de personas jurídicas en el ISOC.

Alberto Atxabal Rada (Atxabal Rada 2016, 304) afirma que las cooperativas poseen unas características propias que las diferencian de las sociedades capitalistas, y que justifican la existencia de un régimen tributario adecuado a sus circunstancias. Entre estas características destaca su función social, su resistencia en contextos de crisis y su contribución a la solución de situaciones precarias de sus socios. Además, este autor destaca dos valores exclusivos de las cooperativas, que las distinguen de las demás formas societarias: la autoayuda o mutualismo, entendida como atención a las necesidades de los socios, y su gestión democrática.

El aspecto mutual también es destacado por Marco Antonio Rodrigo Ruiz (Rodrigo Ruiz 2010, 20), al afirmar que la protección fiscal que reciben las cooperativas está directamente unida al principio mutual presente en dichas entidades. Además, señala este autor que hay otros motivos que justifican la existencia de un régimen especial, como la irrepartibilidad de los fondos sociales, así como los objetivos de tipo económico y social que el cooperativismo persigue, entre los que destaca la participación de los trabajadores en la titularidad de la empresa, democratización de la actividad económica, desarrollo de la inversión productiva y arraigo territorial.

En este mismo sentido, Eva Alonso Rodrigo e Iñaki Santa Cruz Ayo (Alonso Rodrigo y Santa Cruz Ayo 2016, 98), afirman que «el cooperativismo actual ha de plantearse ante el mercado como lo que a menudo es, un modelo de empresa diferente, alternativo al hegemónico capitalista, cuyos rasgos colaboran en la consecución de importantes objetivos sociales de creación de empleo o democratización social, y por ello el sistema fiscal debe compensarlo y apoyarlo reconociéndole un régimen que se adapte a sus especialidades y le ayude a crecer, no a desaparecer».

La función social también es destacada por María del Carmen Pastor del Pino (Pastor del Pino 2016,4), al señalar que los beneficios fiscales previstos en la legislación para las cooperativas tienen su 
fundamento en que promueven el acceso a derechos y bienes constitucionalmente protegidos, como el pleno empleo, el acceso a la vivienda digna, la mejora de la cohesión social y territorial, así como el acceso de distintas clases sociales a los medios de producción y al consumo.

Marina Aguilar Rubio (Aguilar Rubio 2016, 51), defiende la existencia de un régimen tributario especial, para las cooperativas, por el mandato constitucional dirigido a los poderes públicos de fomentar las sociedades cooperativas mediante una legislación adecuada y también por su función social, pues facilitan el acceso de los trabajadores a los medios de producción y promueven la formación de los mismos.

Por otro lado, Aitor Bengoetxea Alkorta (Bengoetxea Alkorta, 1920), considera que el fundamento jurídico del régimen fiscal especial previsto para las cooperativas es doble: por un lado, la misma Constitución Española establece en su artículo 129.2 que los poderes públicos «fomentarán, mediante una legislación adecuada, las sociedades cooperativas»; y por otro, las cooperativas reciben un tratamiento específico de reparto de excedentes, que justifica la existencia de un tratamiento fiscal diferenciado. Así, la legislación contempla la obligación de dotar los fondos de reserva y los fondos para educación y promoción, que no son repartibles. Solo después de cubrir estos dos fondos se pueden distribuir excedentes entre personas socias, como retornos cooperativos. Además, el cálculo del importe a repartir entre las personas socias debe realizarse en función de la actividad cooperativizada que hayan desarrollado, y no en proporción a su aportación al capital.

Por último, Marta Montero Simó (Montero Simó 2016, 19), señala que la existencia de un régimen tributario especial para las cooperativas solo puede fundamentarse en que dichas sociedades poseen diferencias estructurales respecto a las sociedades sujetas al régimen general y que, por tanto, la normativa fiscal de las cooperativas tiene como objetivo adaptar el régimen general a dichas entidades.

El régimen fiscal especial de las cooperativas está recogido, para el Territorio Común, en la Ley 20/1990, de 19 de diciembre, sobre Régimen Fiscal de las Cooperativas (BOE núm. 34, 20 de diciembre de 1990), que derogó la normativa anterior contenida en el Decreto 888/1969, de 9 de mayo, por el que se promulgó el nuevo Estatuto Fiscal de las Cooperativas (BOE núm. 114, de 13 de mayo de 1969).

Juan Francisco Juliá Igual, José Antonio Galindo Bueno y Luis Gallego Sevilla (Juliá Igual, Galindo Bueno y Gallego Sevilla 1999, 150151), destacan varias novedades que introdujo la Ley 20/1990, respecto a la normativa anterior: 
1. Se quiebra el principio mutual como fundamento de la protección fiscal admitiendo que las cooperativas realicen operaciones con terceros, sin que pierdan la condición de fiscalmente protegidas.

2. Se admite la deducibilidad de la dotación obligatoria a reservas que realicen las cooperativas y de los intereses devengados a sus socios, siempre que se encuentren en los límites establecidos por la ley.

3. Se amplía la bonificación de la cuota íntegra en las cooperativas especialmente protegidas, aplicándose no solo sobre la parte de la cuota íntegra correspondiente a los resultados cooperativos, sino también a la parte correspondiente a los resultados extracooperativos.

Por lo que se refiere al País Vasco, la importancia del fenómeno cooperativo y sus peculiaridades hacía recomendable la aprobación de una normativa fiscal específica. Sin embargo, no se contó con una normativa foral propia, reguladora del régimen fiscal de las cooperativas, hasta el año 1997. Como consecuencia de ello, y de acuerdo con lo previsto en la Disposición Adicional Primera de la Ley 12/2002, de 23 de mayo, por la que se aprueba el Concierto Económico con la Comunidad Autónoma del País Vasco, la Ley 20/1990, de 19 de diciembre, sobre el Régimen Fiscal de las Cooperativas, se aplicó, con carácter supletorio, hasta la aprobación en 1997 de las normas forales reguladoras del régimen fiscal de las cooperativas.

Dichas normas forales son las siguientes:

- Norma Foral 2/1997, de 22 de mayo, del Territorio Histórico de Gipuzkoa, sobre Régimen Fiscal de las Cooperativas (BOG núm. 101, de 30 de mayo de 1997).

- Norma Foral 16/1997, de 9 de junio, de las Juntas Generales de Álava, sobre el régimen fiscal de las cooperativas (BOTHA núm. 79, de 18 de junio de 1997).

- Norma Foral 9/1997, de 14 de octubre, del Territorio Histórico de Bizkaia, sobre el Régimen Fiscal de Cooperativas (BOB núm. 211, de 3 de noviembre de 1997), que ha sido sustituida por la Norma Foral 6/2018, de 12 de diciembre, sobre régimen fiscal de cooperativas de Bizkaia (BOB núm. 249, de 28 de diciembre de 2018).

Pero debemos destacar que, tanto la normativa tributaria de Territorio Común como las normas forales indicadas, no establecen un régimen tributario completo para las cooperativas, sino que solo recogen 
una serie de reglas especiales aplicables a las cooperativas, de forma que en lo no previsto se aplicará la normativa recogida con carácter general para el ISOC ${ }^{5}$. Así resulta del número 3 del artículo 1 de la Ley 20/1990 que establece que «en lo no previsto expresamente por esta Ley se aplicarán las normas tributarias generales», e idéntica previsión aparece en el artículo 1 de las normas forales del País Vasco.

\subsection{El régimen de consolidación fiscal en los grupos cooperativos}

Las tres normas forales del País Vasco indicadas anteriormente contienen las especialidades del régimen de consolidación de grupos cooperativos, dentro de su propio articulado. La Norma Foral 2/1997, de 22 de mayo, del Territorio Histórico de Gipuzkoa, y la Norma Foral 16/1997, de 9 de junio, del Territorio Histórico de Álava, en sus artículos 34 a 40, y la Norma Foral 6/2018, de 12 de diciembre, sobre el régimen fiscal de cooperativas de Bizkaia, en sus artículos 44 a 50.

A diferencia de esto, la Ley 20/1990 no contiene una regulación especial del régimen de consolidación fiscal, sino que delega en el Gobierno la regulación de dicho régimen mediante un real decreto. Así, el número tres de la disposición final segunda establece que «El Gobierno, a propuesta del Ministerio de Economía y Hacienda, dictará las normas necesarias para la adaptación de las disposiciones que regulan la tributación sobre el beneficio consolidado de los grupos de Sociedades, contenidas en el Título I del Real Decreto-Ley 15/1977, de 25 de febrero ( $B O E$ núm. 50, de 28 de febrero de 1977), y en el Real Decreto 1414/1977, de 17 de junio (BOE núm. 150, de 24 de junio de 1977), a las especialidades de las Sociedades Cooperativas que en virtud de sus reglas estatutarias mantengan relaciones de vinculación en el ejercicio de sus actividades empresariales».

En ejecución de esta delegación, el Gobierno aprobó el Real Decreto 1345/1992, de 6 de noviembre, por el que se dictan normas para la adaptación de las disposiciones que regulan la tributación sobre el

5 En Territorio Común, la normativa reguladora del ISOC se encuentra recogida la Ley 27/2014, de 27 de noviembre, del Impuesto sobre Sociedades (BOE núm. 288. De 28 de noviembre de 2014), y en el País Vasco, en la Norma Foral 11/2013, de 5 de diciembre, del Impuesto sobre Sociedades de Bizkaia (BOB núm. 238, de 13 de diciembre de 2013), en la Norma Foral 37/2013, de 13 de diciembre, del Impuesto sobre Sociedades de Álava (BOTHA núm. 148, de 27 de diciembre de 2013) y en la Norma Foral 2/2014, de 17 de enero, del Impuesto sobre Sociedades del Territorio Histórico de Gipuzkoa (BOG núm. 13, de 22 de enero de 2014). 
beneficio consolidado a los grupos de sociedades cooperativas ( $B O E$ núm. 292, de 5 de diciembre de 1992).

Por otro lado, si antes hemos señalado que tanto la Ley 20/1990 como las normas forales reguladoras del régimen fiscal de las cooperativas, no recogen un régimen completo aplicable a las mismas, tampoco las normas reguladoras del régimen de consolidación de los grupos cooperativos son completas, por lo que, también en este ámbito, la normativa reguladora del ISOC se aplicará con carácter supletorio a los grupos cooperativos, en todo lo que no se regule por su normativa especial.

Las diferencias entre el régimen de consolidación previsto en la normativa del ISOC y el régimen de consolidación recogido en la normativa tributaria de las cooperativas se centran principalmente en la definición de grupo de cooperativas y en la determinación de un mecanismo de consolidación adecuado a las características especiales de las mismas.

\subsubsection{DeFINICIÓN DE GRUPO DE SOCIEDADES COOPERATIVAS}

Debemos destacar, en primer lugar, que no hay diferencias entre la normativa tributaria de Territorio Común y del País Vasco al elaborar un concepto de grupo cooperativo que pueda optar por aplicar el régimen especial de consolidación fiscal.

A los efectos del régimen de declaración consolidada, se entenderá por grupo cooperativo el conjunto formado por una entidad cabeza de grupo y las cooperativas que tengan la condición de socia o asociada de aquella sobre las que ejerza poderes de decisión, en virtud de sus reglas estatutarias.

La entidad cabeza del grupo de sociedades cooperativas será una sociedad cooperativa o cualquier otra entidad siempre que, en este último caso, su objeto exclusivo sea el de planificar y coordinar el desarrollo empresarial y las estrategias a largo plazo de las cooperativas que integran el grupo, no pudiendo estar participada por otras personas o entidades diferentes a estas últimas ${ }^{6}$.

6 Esta definición está recogida en el artículo 1.2 del Real Decreto 1345/1992, de 6 de noviembre, por el que se dictan normas para la adaptación de las disposiciones que regulan la tributación sobre el beneficio consolidado a los grupos de sociedades cooperativas; en el artículo 34.2 de la Norma Foral 2/1997, de 22 de mayo, del Territorio Histórico de Gipuzkoa; en el artículo 34.2 de la Norma Foral 16/1997, de 9 de junio, del Territorio Histórico de Álava, y en el artículo 44.2 de la Norma Foral 6/2018, de 12 de diciembre, sobre régimen fiscal de cooperativas de Bizkaia. 
Hasta aquí, la definición de grupo cooperativo desde el punto de vista fiscal coincide con el concepto mercantil.

Sin embargo, la normativa tributaria establece un requisito adicional, no previsto en la legislación mercantil, para que el grupo cooperativo pueda acogerse al régimen de consolidación fiscal: debe existir, entre las cooperativas del grupo, un compromiso de redistribuir solidariamente el excedente neto obtenido por cada una de las cooperativas integrantes del grupo, el cual deberá constar en escritura pública suscrita por todas ellas, así como en sus respectivos estatutos.

La redistribución afectará, como mínimo, al 25 por ciento del excedente neto, una vez deducidos los impuestos y las cantidades destinadas por imperativo de la Ley a los fondos de reserva, y deberá realizarse en forma directamente proporcional al importe económico de las operaciones, actividades y servicios cooperativizados realizados por los socios de las cooperativas, miembros del grupo.

Este compromiso de redistribución solidario no aparece en la legislación mercantil por lo que podemos entenderlo como un requisito adicional, que los grupos cooperativos deben atender para poder acogerse al régimen de consolidación.

Por otro lado, la normativa del ISOC establece dos requisitos para la aplicación del régimen de consolidación, que no se recogen en la normativa tributaria de las cooperativas, y que no serán aplicables a los grupos cooperativos, por sus especiales características:

1. El primero se refiere a la forma que deben adoptar las entidades integrantes de un grupo, que es la de sociedad anónima, de responsabilidad limitada, o comanditaria por acciones, admitiéndose también que puedan integrarse en el grupo los establecimientos permanentes de entidades no residentes. Lógicamente este requisito formal no puede aplicarse a los grupos cooperativos, pues estos deben estar formados, al menos en su base, por cooperativas.

2. El segundo de los requisitos consiste en que la entidad dominante debe tener una participación, directa o indirecta, de al menos el 75 por ciento del capital social, y poseer la mayoría de los derechos de voto de las entidades del grupo que sean dependientes, el primer día del período impositivo en que sea de aplicación el régimen especial, y que dicha participación y los referidos derechos de voto se mantengan durante todo el período impositivo, salvo en el supuesto de disolución de la entidad participada. El porcentaje anterior se reducirá al 70 por ciento del capital social, si se trata de entidades cuyas accio- 
nes estén admitidas a negociación en un mercado regulado, o cuando se tengan participaciones indirectas en otras entidades, siempre que dicho porcentaje se alcance a través de entidades participadas, cuyas acciones estén admitidas a negociación en un mercado regulado. Como hemos indicado anteriormente, los grupos de cooperativas no se basan en la idea de control sino en la unidad de decisión, por lo que no tendría sentido exigir este requisito a los grupos cooperativos, previsto en la normativa del ISOC. Como afirma Marina Aguilar Rubio (Aguilar Rubio 2017, 127), la relación de dominio en un grupo cooperativo tiene "una dirección inversa» frente a los grupos que aplican el régimen de consolidación fiscal previsto en la normativa del ISOC. Si en estos últimos, el poder de decisión de la sociedad dominante sobre las sociedades dependientes deriva de la participación que la primera tiene en el capital de las segundas, en un grupo cooperativo es la entidad cabeza del grupo la que está participada por las cooperativas que lo integran, de forma que el control sobre estas no es consecuencia de una participación en el capital, sino de los acuerdos existentes entre las entidades miembros del grupo.

A pesar de estas diferencias existentes entre el régimen de consolidación del ISOC y el régimen de consolidación de los grupos cooperativos, hay que destacar que ambos regímenes también comparten algunos elementos, entre los que destaca el ser un régimen opcional.

De este modo, los grupos cooperativos podrán aplicar dos regímenes de tributación:

1. El régimen de tributación individual, como si no existiera dicho grupo. En este caso cada sociedad del grupo contabiliza su gasto por ISOC en función de su excedente cooperativo y teniendo en cuenta las posibles diferencias permanentes que puedan resultar de sus operaciones.

2. El régimen de tributación consolidada, cuando las cooperativas del grupo, en virtud de sus reglas estatutarias, mantengan relaciones de vinculación en el ejercicio de sus actividades empresariales. En este caso el sujeto pasivo del impuesto es el propio grupo, ostentando la entidad cabeza de grupo las «facultades, responsabilidades y obligaciones» establecidas en la normativa del ISOC para la sociedad dominante del grupo de sociedades. Es decir, la entidad cabeza del grupo será la responsable de la gestión, liquidación y recaudación del impuesto, pero las entidades que integran el grupo están igualmente sujetas a las obli- 
gaciones tributarias que se deriven del régimen de tributación individual, excepción hecha del pago de la deuda, y responden solidariamente del pago de la deuda tributaria consolidada, excluidas las sanciones.

Como consecuencia del carácter opcional de este régimen especial, es la entidad cabeza de grupo la que solicitará a la Administración tributaria la aplicación del mismo ${ }^{7}$, a cuyo efecto presentará la siguiente documentación:

a) Acuerdo de las Asambleas Generales de las sociedades cooperativas en el que manifiestan su voluntad de acogerse al régimen de tributación sobre el beneficio consolidado.

b) Escritura pública en la que conste el compromiso de redistribución solidaria.

c) Estatutos de las sociedades cooperativas pertenecientes al grupo.

Las sociedades cooperativas que con posterioridad a la concesión del régimen de declaración consolidada reúnan los requisitos expuestos anteriormente, quedarán incluidas obligatoriamente en el grupo de sociedades cooperativas, con efecto desde el ejercicio siguiente a aquél en que concurran los mismos. Estos requisitos son la existencia de una vinculación en el ejercicio de sus actividades empresariales, en virtud de sus reglas estatutarias, de forma que la cooperativa que se integra en el grupo quedará sujeta a los poderes de decisión de la entidad cabeza del mismo. Esta vinculación comprenderá el compromiso de redistribuir solidariamente el 25 por ciento del excedente neto, tal y como hemos indicado anteriormente.

Por el contrario, se excluirán del grupo las sociedades cooperativas que dejasen de reunir los requisitos, con efecto desde el mismo ejercicio en que tal circunstancia se produzca.

En cuanto a la pérdida del régimen especial se establece que, además de las causas previstas en la normativa general, el régimen de declaración consolidada se perderá a causa del incumplimiento del

7 La documentación que debe presentarse ante la Administración tributaria está recogida en el artículo 2.1 del Real Decreto 1345/1992, de 6 de noviembre, por el que se dictan normas para la adaptación de las disposiciones que regulan la tributación sobre el beneficio consolidado a los grupos de sociedades cooperativas; en el artículo 35.1 de la Norma Foral 2/1997, de 22 de mayo, del Territorio Histórico de Gipuzkoa; en el artículo 35.1 de la Norma Foral 16/1997, de 9 de junio, del Territorio Histórico de Álava, y en el artículo 45.1 de la Norma Foral 6/2018, de 12 de diciembre, sobre régimen fiscal de cooperativas de Bizkaia. 
compromiso de redistribuir solidariamente el excedente cooperativo disponible.

Así mismo, será causa de pérdida del régimen de declaración consolidada, la realización por la entidad cabeza de grupo, cuando no sea sociedad cooperativa, de actividades no comprendidas dentro del objeto exclusivo de planificar y coordinar el desarrollo empresarial y las estrategias a largo plazo de las cooperativas que integran el grupo.

\subsubsection{LA DETERMINACIÓN DE LA BASE/CUOTA CONSOLIDADA}

Debemos partir de que la normativa del ISOC ${ }^{8}$, establece un sistema de consolidación de bases imponibles de las sociedades integrantes del grupo fiscal. Dado que el grupo de sociedades, a efectos fiscales, es considerado como una unidad económica, las rentas obtenidas por el grupo provienen exclusivamente de las operaciones realizadas por él mismo con terceros. Ello implica que los resultados procedentes de operaciones efectuadas entre sociedades del grupo, en el período impositivo, deben ser eliminados al tiempo de determinar la base imponible consolidada, y posteriormente se incorporarán a la base imponible del grupo fiscal, cuando se realicen frente a terceros.

Estas eliminaciones e incorporaciones de resultados se realizan teniendo en cuenta los criterios que están establecidos en la normativa contable sobre formulación de cuentas anuales consolidadas.

La eliminación afectará a las operaciones internas, entre las que podemos diferenciar:

a) Gastos e ingresos por operaciones internas.

b) Existencias.

c) Inmovilizado.

d) Servicios.

e) Activos financieros.

f) Pérdida por deterioro del valor de la participación.

g) Dividendos internos (salvo en el supuesto previsto en el artículo 33.3 de la Ley del ISOC).

8 El sistema de consolidación de bases imponibles está regulado en el artículo 62 y siguientes de la Ley 27/2014, de 27 de noviembre, del Impuesto sobre Sociedades; en el artículo 89 y siguientes de la Norma Foral 11/2013, de 5 de diciembre, del Impuesto sobre Sociedades de Bizkaia; en el artículo 89 y siguientes de la Norma Foral 37/2013, de 13 de diciembre, del Impuesto sobre Sociedades de Álava; y en el artículo 89 y siguientes de la Norma Foral 2/2014, de 17 de enero, del Impuesto sobre Sociedades del Territorio Histórico de Gipuzkoa. 
Frente a este régimen, los grupos cooperativos que hayan optado por acogerse al régimen de consolidación aplicarán un mecanismo de consolidación diferente, según estén sujetos a la normativa de Territorio Común o a la normativa foral del País Vasco.

Los grupos de cooperativas sujetos a la normativa de Territorio Común, que hayan optado por aplicar el régimen de consolidación fiscal, no aplican un sistema de consolidación de bases imponibles, sino que, tal y como señalan los artículos 3 y 4 del Real Decreto 1345/1992, cada una de las entidades del grupo calculan la suya de forma separada, pudiendo ser positiva o negativa. Una vez calculada la base individual, lo que se consolida son las cuotas.

Según Tomás Garrido Pulido, Raquel Puentes Poyatos y Emilio Navarro Heras (Garrido Pulido, Puentes Poyatos y Navarro Heras 2007, 79), la razón de este diferente tratamiento se justifica en la existencia, entre las diferentes clases de cooperativas, de tipos tributarios distintos, por lo que aplicar un sistema de consolidación de bases haría necesario elegir un único tipo tributario a aplicar. Además, en las cooperativas sujetas a la normativa de Territorio Común, se distinguen dos bases imponibles, una formada por los resultados cooperativos y otra formada por los resultados extracooperativos, con diferentes tipos impositivos.

En definitiva, cada cooperativa integrante del grupo determina su base imponible individual realizando exclusivamente las eliminaciones por:

a) Retornos entre sociedades cooperativas del grupo.

b) Las ayudas económicas que, en cumplimiento de las obligaciones asumidas, deban prestarse entre sí las sociedades cooperativas del grupo.

c) Resultados distribuidos por la entidad cabeza de grupo.

Por tanto, el resto de operaciones internas del grupo no pueden ser eliminadas.

Una vez determinada la base imponible, cada entidad del grupo obtendrá su cuota, positiva o negativa, aplicando a las bases imponibles positivas o negativas, los tipos de gravamen que correspondan, así como las bonificaciones que resulten aplicables.

A continuación, se obtendrá la cuota íntegra consolidada mediante la suma de las cuotas tributarias individuales de cada una de las entidades del grupo. Si la suma resultase negativa, su importe podrá compensarse con las cuotas íntegras positivas de los períodos impositivos siguientes, con el mayor de los siguientes límites:

a) El 70 por ciento de la cuota íntegra, previa a su compensación. 
b) El importe que resulte de multiplicar un millón de euros por el tipo medio de gravamen de la entidad.

Las cuotas tributarias negativas de cualquier sociedad cooperativa que se hubieran producido en períodos impositivos anteriores a aquél en que dicha cooperativa tribute en régimen de declaración consolidada, serán compensables solo con las cuotas tributarias positivas de la propia sociedad cooperativa y hasta el límite de estas.

Frente a este régimen, recogido en el RD 1345/1992 para el Territorio Común, los grupos de cooperativas sujetos a la normativa foral que hayan optado por aplicar el régimen de consolidación fiscal utilizan un sistema de consolidación de bases, similar al previsto en la normativa del ISOC, pero con algunas especialidades.

El fundamento de que, en el País Vasco, se sustituyera, en el año 1997, el sistema de compensación de cuotas por el sistema de compensación de bases se encuentra, según la exposición de motivos de las tres normas forales de 1997, en que dichas normas eliminaron la diferenciación entre resultados cooperativos y extracooperativos. Además, se introdujo un sistema de tipo único, que equiparaba la estructura de liquidación del impuesto a la estructura general del ISOC.

Sin embargo, este sistema de consolidación de bases imponibles presenta una importante diferencia con el previsto en la normativa del ISOC.

Así, se establece ${ }^{9}$ que para la determinación de la base imponible consolidada se realizarán exclusivamente las eliminaciones por operaciones intergrupo que procedan de:

a) Retornos entre sociedades cooperativas del grupo.

b) Las ayudas económicas que, en cumplimiento de las obligaciones asumidas, deban prestarse entre sí las sociedades cooperativas del grupo.

c) Resultados distribuidos por la entidad cabeza de grupo.

En cuanto al tipo aplicable a la base consolidada, la normativa foral establece, para las cooperativas protegidas, un tipo general del 20 por ciento. Para las cooperativas especialmente protegidas, además de este tipo del 20 por ciento, se establece una bonificación del 50 por ciento de la cuota líquida. Y en el supuesto de que en un mismo grupo coe-

9 En el artículo 36 de la Norma Foral 2/1997, de 22 de mayo, del Territorio Histórico de Gipuzkoa; en el artículo 36 de la Norma Foral 16/1997, de 9 de junio, del Territorio Histórico de Álava; y en el artículo 46 de la Norma Foral 6/2018, de 12 de diciembre, sobre el régimen fiscal de cooperativas de Bizkaia. 
xistan cooperativas protegidas y especialmente protegidas la bonificación se aplicará exclusivamente, sobre la cuota líquida correspondiente a los resultados procedentes de las operaciones realizadas con las cooperativas especialmente protegidas.

La cuota íntegra y la cuota líquida del grupo de sociedades cooperativas se minorarán en el importe de las deducciones previstas, tanto en la normativa general del ISOC como en la normativa tributaria de las cooperativas. Los requisitos establecidos para disfrutar de las mencionadas deducciones se referirán al grupo de sociedades cooperativas.

Por último, las deducciones de cualquier cooperativa pendientes de deducir en el momento de su inclusión en el grupo de sociedades cooperativas podrán deducirse en la cuota íntegra o de la cuota líquida del mismo, según corresponda, con el límite que hubiere correspondido a aquélla en el régimen individual de tributación.

\section{Conclusiones}

PRIMERA. En relación con el concepto de grupo de sociedades, prácticamente la totalidad de la doctrina defiende que el artículo 42 CCo contiene un concepto general de grupo aplicable a todos los ámbitos del derecho mercantil. Este artículo consagra, como elemento esencial del grupo de sociedades, el control que la sociedad dominante ejerce sobre las demás sociedades integrantes del grupo, a las que llamamos dependientes o subordinadas.

Frente a esto, el concepto de grupo cooperativo prescinde de la idea de control, como elemento configurador del mismo. Así, en el grupo cooperativo, la entidad cabeza de grupo ejerce la dirección del mismo, emitiendo instrucciones de obligado cumplimiento, pero esto no deriva del control ejercido sobre el resto de los miembros del grupo sino del traspaso de poderes o competencias que las cooperativas integrantes del grupo realizan en favor de la entidad cabeza del mismo, en el momento de la constitución del grupo. De hecho, en un grupo cooperativo, el control no lo ejerce la entidad cabeza sino las cooperativas que están en la base, que son las que poseen el capital de la entidad cabeza y las que determinan, mediante sus acuerdos, cómo debe ejercerse la dirección unitaria.

Estas diferencias esenciales van a dar lugar a que, en los grupos cooperativos que opten por aplicar el régimen de consolidación, no se requiera la participación de la entidad cabeza de grupo en más de un 75 por ciento del capital de las cooperativas integrantes. Éste sería un requisito de imposible cumplimiento ya que la entidad cabeza del 
grupo no tiene participaciones en las cooperativas integrantes, sino que son estas las que tienen la titularidad del capital de la primera.

SEGUNDA. Por lo que se refiere al procedimiento de consolidación en el ámbito del Territorio Común, la especialidad más importante recogida en la normativa tributaria de las cooperativas en relación a la normativa del ISOC, consiste en que se sustituye el sistema de consolidación de bases del ISOC por un sistema de consolidación de cuotas, de forma que cada cooperativa integrante del grupo determinará su base imponible de forma independiente, y será la suma de cuotas de cada cooperativa lo que se consolidará. El fundamento de esta diferencia se basa en la distinción, dentro de los resultados de cada cooperativa, entre beneficios cooperativos y extracooperativos, que son objeto de un tipo impositivo distinto, y a su vez, en la existencia de diversos tipos impositivos aplicables a las cooperativas, según su actividad.

Además, para la determinación de las bases individuales no se contempla la eliminación de todas las operaciones intragrupo, sino exclusivamente de los retornos entre las cooperativas del grupo, de las ayudas económicas que las cooperativas deban prestarse entre sí y de los resultados distribuidos por la entidad cabeza del grupo.

TERCERA. Por su parte, en el ámbito del País Vasco, la normativa tributaria no recoge este sistema especial de consolidación, sino que mantiene el procedimiento de consolidación de bases previsto en el ISOC, fundamentándose esta opción, en la normativa de 1997, en la eliminación de la diferenciación entre resultados cooperativos y extra cooperativos y en la unificación de los tipos impositivos aplicables a las cooperativas sujetas a la normativa foral vasca, que equiparaba la estructura de liquidación del impuesto a la estructura general del ISOC.

\section{Bibliografía}

AGUILAR RUBIO, Marina. 2016. «El régimen fiscal de las cooperativas y el Derecho de la Unión Europea». Boletín de la Asociación Internacional de Derecho Cooperativo, n. ${ }^{\circ}$ 50: 49-71. https://doi.org/10.18543/baidc-502016pp49-71.

AGUILAR RUBIO, Marina. 2017. "Análisis crítico de la fiscalidad de las cooperativas de segundo grado como fórmula de integración». REVESCO. Revista de Estudios Cooperativos, monográfico 126: 118-132.

ALFONSO SÁNCHEZ, Rosalía y Mercedes Sánchez Ruiz. 2007. "Aspectos generales sobre el buen gobierno de las cooperativas y de los grupos cooperativos». Revista de Derecho Mercantil, n. ${ }^{\circ} 266$. 
ALONSO RODRIGO, Eva e Iñaki Santa Cruz Ayo. 2016. "Identidad, competitividad y creación de empleo». Boletín de la Asociación Internacional de Derecho Cooperativo, n. ${ }^{\circ}$ 50: 73-101. https://doi.org/10.18543/baidc-502016pp73-101.

ATXABAL RADA, Alberto. 2016. "La identidad cooperativa como justificación de un tratamiento diferenciado». Boletín de la Asociación Internacional de Derecho Cooperativo, n. ${ }^{\circ}$ 50: 285-307. https://doi.org/10.18543/baidc-502016pp285-307.

ATXABAL RADA, Alberto. 2018. "Los impuestos personales sobre el capital en las cooperativas y en sus socios en el País Vasco». Boletín de la Asociación Internacional de Derecho Cooperativo, n. ${ }^{\circ} 52$ (julio), 137-66. https://doi. org/10.18543/baidc-52-2018pp137-166.

ATXABAL RADA, Alberto. 2020. «Las medidas fiscales para favorecer el emprendimiento por las cooperativas». REVESCO. Revista de Estudios Cooperativos 133, n. ${ }^{\circ}$ abril: e67335. https://doi.org/10.5209/reve.67335

BENGOETXEA ALKORTA, Aitor. 2016. "Las cooperativas». CIRIEC-España. Revista Jurídica, núm. 29: 1-30.

CASTRO DE LUNA, Manuel José. 2015. «Hacia un modelo contractual del concepto de grupo de sociedades». Revista Quincena Fiscal, n. 20.

EMBID IRUJO, José Miguel.2012. "Ante la regulación de los grupos de sociedades en España». Revista de Derecho Mercantil, n. 284.

GARCÍA OREJUDO, Raúl. 2012. "Concursos conexos a la luz de la reforma» en Pedro Martín Molina, Emilio González Bilbao y José María del Carre DíezGálvez (Coord.). La reforma de la ley concursal analizada por especialistas. Madrid: Dykinson.

GARRIDO PULIDO, Tomás, Raquel Puentes Poyatos y Emilio Navarro Heras. 2007. "Fiscalidad de las sociedades cooperativas de segundo grado». REVESCO. Revista de Estudios Cooperativos, n. ${ }^{\circ}$ 91: 60-92.

GONDRA ELGUEZABAL, Gotzon. 2021. «La contribución para la educación y promoción cooperativa y otros fines de interés público - COFIP - en la ley vasca y su aplicabilidad». Boletín de la Asociación Internacional de Derecho Cooperativo, n. ${ }^{\circ} 58$ (mayo), 137-173. https://doi.org/10.18543/ baidc-58-2021 pp137-173.

JULIÁ IGUAL, Juan Francisco, José Antonio Galindo Bueno y Luis Gallego Sevilla. 1999. "Normativa central y autonómica de la empresa cooperativa en España. Especial referencia a su Régimen Económico y Fiscal». REVESCO. Revista de Estudios Cooperativos, n. ${ }^{\circ}$ 67: 139-174.

LÓPEZ APARCERO, Antonio. 2012. "Concepto de grupo de sociedades y concurso». Anuario de Derecho Concursal, n. 26.

MONTERO SIMÓ, Marta. 2016. "La fiscalidad de las cooperativas ante el nuevo Impuesto sobre Sociedades». Boletín de la Asociación Internacional de Derecho Cooperativo, n. ${ }^{\circ}$ 50: 17-47. https://doi.org/10.18543/baidc50-2016pp17-47.

NAGORE, Iñigo. 2000. "Grupos y Asociaciones Cooperativas». Boletín de la Asociación Internacional de Derecho Cooperativo. La Ley General de Cooperativas de España, n. ${ }^{\circ}$ 34: 115-146. 
PASTOR DEL PINO, María del Carmen. 2016. "Las cooperativas como sujetos de protección fiscal». CIRIEC- España. Revista Jurídica, n. ${ }^{\circ} 28:$ 1-33.

RAMOS MUÑOZ, David. 2015. «El Derecho de grupos que viene. Cuidado con lo que deseas». En: Estudios sobre el futuro Código Mercantil: libro homenaje al profesor Rafael IIlescas Ortiz. Getafe: Universidad Carlos III de Madrid.

RODRIGO RUIZ, Marco Antonio. 2010. «Reflexiones sobre la reforma de la fiscalidad de las cooperativas y sobre su compatibilidad con el Derecho comunitario». GEZKI, n. ${ }^{\circ}$ 6: 7-28.

ROJO FERNÁNDEZ-RÍO, Ángel. 1996. "Los grupos de sociedades en el Derecho Español». Revista de Derecho Mercantil, n. ${ }^{\circ} 220$.

VARGAS VASSEROT, Carlos, Enrique Gadea Soler y Fernando Sacristán Bergia. 2017. Derecho de las sociedades cooperativas. Régimen económico, integración, modificaciones estructurales y disolución. Madrid: Wolters Kluwer España. 


\section{Derechos de autor}

El Boletín de la Asociación Internacional de Derecho Cooperativo es una revista de acceso abierto lo que significa que es de libre acceso en su integridad inmediatamente después de la publicación de cada número. Se permite su lectura, la búsqueda, descarga, distribución y reutilización legal en cualquier tipo de soporte sólo para fines no comerciales y según lo previsto por la ley; sin la previa autorización de la Editorial (Universidad de Deusto) o el autor, siempre que la obra original sea debidamente citada (número, año, páginas y DOI si procede) y cualquier cambio en el original esté claramente indicado.

\section{Copyright}

The International Association of Cooperative Law Journal is an Open Access journal which means that it is free for full and immediate access, reading, search, download, distribution, and lawful reuse in any medium only for non-commercial purposes, without prior permission from the Publisher or the author; provided the original work is properly cited and any changes to the original are clearly indicated. 